\title{
Die verschleierte Konzernrichtlinie
}

\section{Zu den neuen EU-Vorgaben für related party transactions und ihren Auswirkungen auf das deutsche Recht}

\author{
Andreas Tarde, Heidelberg*
}

\author{
Inhaltsübersicht
}

ZGR 2017, 360-388

I. Die Reform der Aktionärsrechterichtlinie kommt . . . . . . . . . . . . . . . . . 361

II. Überblick zu Art. 9c ARRL II . . . . . . . . . . . . . . . . . . . . . . . . 362

1. Anwendungsbereich . . . . . . . . . . . . . . . . 362

2. Verfahrenspflichten . . . . . . . . . . . . . . . . . 366

3. Bereichsausnahmen ....................... 370

4. Sanktionen . . . . . . . . . . . . . . . . . . . 372

III. Umsetzungsperspektiven in Deutschland . . . . . . . . . . . . . . . . . . . . 372

1. Notwendigkeit einer vollständigen RPT-Definition . . . . . . . . . . . . . 373

2. Schlüsselfunktion der Wesentlichkeitsschwelle . . . . . . . . . . . . . . . 374

3. RPT-Aggregation als Bürokratiemonster im Konzern . . . . . . . . . . . . . 376

4. Sinnvolle Ergänzung bestehender Publizitätspflichten . . . . . . . . . . . . . . 377

5. Fehlende Notwendigkeit einer Fairness Opinion . . . . . . . . . . . . . . . 378

6. Zustimmungsvorbehalt des Aufsichtsrats bzw. Related-Party-Ausschusses 379

7. Keine Berücksichtigung des Gruppeninteresses, Abschaffung des gestreckten Nachteilsausgleichs . . . . . . . . . . . . . . . . . . . . 382

8. Gelockerte Publizitätspflichten für RPT der Tochtergesellschaften . . . . . 383

9. Ausschöpfung der optionalen Bereichsausnahmen, fehlende Konzernaus-

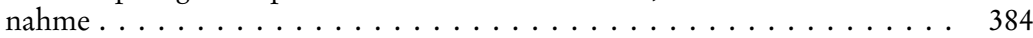

10. Keine Anwendung auf geschlossene Gesellschaften . . . . . . . . . . . 386

IV. Fazit und Ausblick . . . . . . . . . . . . . . . . . . . . . . . 386

In Kürze wird die Änderung der Aktionärsrechterichtlinie (2007/36/EG) in Kraft treten. Mit ibrer Umsetzung werden erstmals auch abseits von Rechnungslegungs- und Prospektpflichten Spezialvorschriften für sogenannte related party transactions in das deutsche Recht eingeführt werden. Der vorliegende Beitrag untersucht die kommende RPT-Regelung in Art. 9c der reformierten Aktionärsrechterichtlinie und ihre Umsetzungsperspektiven mit Blick auf die Folgen für Unternehmensgruppen. Wie sich zeigt, kommen auf die Praxis deutscher Konzerne trotz mancher Nachbesserung am ursprünglichen Vorschlag der EU-Kommission erbebliche Umwälzungen zu.

The amendment of the Shareholder Rights Directive (2007/36/EC) is set to come into force soon. To transpose the revised directive into German law, it will be necessary to establish special provisions for related party transactions that go far beyond existing accounting and prospectus requirements. This article examines the upcoming regulations of related party transactions in Art. $9 c$ of the amended Shareholder Rights Directive and their prospective implementation into German law

* Der Autor ist wiss. Hilfskraft und Doktorand bei Prof. Dr. Dr. h.c. mult. Peter Hommelhoff am Institut für deutsches und europäisches Gesellschafts- und Wirtschaftsrecht der Ruprecht-Karls-Universität Heidelberg. 
with regard to the effects on corporate groups. In comparison with the initial proposal by the European Commission, many improvements have been made in the final version of the amending directive. However, as the article shows, corporate groups in Germany will still have to face significant changes in their business activities.

\section{Die Reform der Aktionärsrechterichtlinie kommt}

Mitte Dezember 2016 haben sich die Vertreter von Kommission, Rat und Parlament auf einen Kompromisstext zur Änderung der Aktionärsrechterichtlinie geeinigt. ${ }^{1}$ Nachdem sowohl das Parlament als auch der Rat die vereinbarte Fassung am 14. März bzw. 3. April 2017 angenommen haben, wird die Änderungsrichtlinie nach dreijährigem Gesetzgebungsprozess nun in Kürze in Kraft treten und von den Mitgliedstaaten innerhalb von 24 Monaten umgesetzt werden müssen. ${ }^{2}$

Art. 9c der reformierten Aktionärsrechterichtlinie („ARRL II“) wird Regelungen für Geschäfte zwischen einer Gesellschaft und ihr nahestehenden Unternehmen und Personen einführen, ein Geschäftstyp, für den sich im angelsächsischen Raum der griffige Terminus related party transactions („RPT“) etabliert hat. Damit sollen Gesellschaften und ihre Minderheitsaktionäre vor jenen Geschäften geschützt werden, die im internationalen Schrifttum als typisches Ausbeutungsinstrument einflussreicher Insider ausgemacht wurden. ${ }^{3}$

Viel war im Vorfeld über die Unausgegorenheit des Kommissionsvorschlags zum neuen Art. 9c ARRL II ${ }^{4}$ berichtet worden. ${ }^{5}$ Der auf einer Empfehlung des European Corporate Governance Forum ${ }^{6}$ beruhende Entwurf entsprach

1 Dok. 15248/16 vom 13.12.2016, abrufbar unter http://data.consilium.europa.eu/doc/document/ST-15248-2016-INIT/en/pdf.

2 Richtlinie des Europäischen Parlaments und des Rates zur Änderung der Richtlinie 2007/ 36/EG im Hinblick auf die Förderung der langfristigen Mitwirkung der Aktionäre, Art. 3 Abs. 1; die Veröffentlichung im Amtsblatt der EU stand bei Redaktionsschluss noch aus.

3 Vgl. Enriques, EBOR 2015, 1, 2; Djankov/La Porta/Lopez-De-Silanes/Shleifer/ Vishny, J. Financ. Econ. 88 (2008), 430, 462; Johnson/La Porta/Lopez-De-Silanes/ Shleifer, Am. Econ. Rev. 90 (2000), $22 \mathrm{f}$.

4 Vorschlag für eine Richtlinie des Europäischen Parlaments und des Rates zur Änderung der Richtlinie 2007/36/EG im Hinblick auf die Förderung der langfristigen Einbeziehung der Aktionäre sowie der Richtlinie 2013/34/EU in Bezug auf bestimmte Elemente der Erklärung zur Unternehmensführung, COM/2014/0213 final.

5 Vgl. etwa Bremer, NZG 2014, 415; Drygala, AG 2013, 198, $207 \mathrm{ff}$; Enriques, EBOR 2015, 1, 25 ff; Lanfermann/Maul, BB 2014, 1283, 1287f; SeibT, DB 2014, 1910, $1914 \mathrm{ff}$; SimON, DB 4/2015, M5.

6 Statement of the European Corporate Governance Forum on Related Party Transactions for Listed Entities vom 10. März 2011, abrufbar unter http://ec.europa.eu/internal_market/company/docs/ecgforum/ecgf_related_party_transactions_en.pdf. 
im Wesentlichen dem Regelungsansatz der britischen Listing Rules ${ }^{7}$, ohne jedoch deren Bereichsausnahmen zu übernehmen oder Spielräume für eine Anpassung an abweichende Rahmenbedingungen in anderen Mitgliedstaaten $\mathrm{zu}$ enthalten. Im Laufe der Verhandlungen zwischen Kommission, Rat und Parlament musste die Norm daher grundlegend überarbeitet werden. Die verabschiedete Fassung des Art. 9c ARRL II lässt den Mitgliedstaaten nun eine Reihe von Wahloptionen. Die Sorge, Deutschland müsse eine hierzulande "allen Erfahrungen von guter Corporate Governance" ${ }^{\text {"8 }}$ widersprechende RPT-Regelung einführen, ist genommen.

Im Konzern gehören die verbundenen Mutter-, Tochter- und Schwestergesellschaften einer Gesellschaft nicht selten zum Kreis ihrer wichtigsten Geschäftspartner. Ausfluss einer effizienten Binnenintegration der Gruppe können dann zahlreiche related party transactions zwischen den einzelnen Konzerngesellschaften sein. Im deutschen Schrifttum wurde daher früh die große Bedeutung der geplanten Regelungen für Unternehmensgruppen erkannt. ${ }^{9}$ Erste Entwürfe ließen befürchten, dass die neue Richtlinie die $\$ \mathbb{S} 291 \mathrm{ff}$ AktG weitgehend obsolet machen und Konzernen einen kaum zu bewältigenden Verwaltungsmehraufwand bringen würde. ${ }^{10}$ Auch in seiner endgültigen Fassung ist Art. 9c ARRL II eine Norm, die starke Überschneidungen mit konzernrechtlichen Regelungen aufweist und Konzerne in besonderer Weise betrifft. Im Folgenden werden die neuen Regelungen untersucht und ihre mögliche Umsetzung in Deutschland mit Blick auf die Auswirkungen auf Unternehmensgruppen und das sie regelnde Konzernrecht erörtert.

\section{II. Überblick zu Art. 9c ARRL II}

\section{Anwendungsbereich}

Art. 9c ARRL II gilt für alle wesentlichen Transaktionen einer Gesellschaft mit nahestehenden Unternehmen und Personen.

7 Siehe FCA Handbook LR 11.1.

8 So U. H. SCHNEIDER, EuZW 2014, 641, 642 zum Kommissionsvorschlag.

9 Vgl. Drygala, AG 2013, 198, 206; Hommelhoff, KSzW 2014, 63, 66f; Hopt, ZGR 2013, 165, 210; SЕıвт, Börsen-Zeitung, 28.6.2014, 9 („Frontalangriff auf deutsches Konzernrecht").

10 Vgl. Drygala, AG 2013, 198, $206 \mathrm{ff}$. 


\section{a) Börsengesellschaften, Art. 1 Abs. 1 ARRL II}

Auch in ihrer reformierten Fassung wird die Aktionärsrechterichtlinie gemäß ihrem Art. 1 Abs. 1 nur für Gesellschaften gelten, deren Aktien zum Handel an einem geregelten Markt in einem Mitgliedstaat zugelassen sind. Der weitergehende Vorschlag des Rechtsausschusses des EU-Parlaments ${ }^{11}$, große Unternehmen und Gruppen im Sinne der Bilanzrichtlinie (2013/34/EU) einzubeziehen, wurde im Gesetzgebungsverfahren rasch wieder aufgegeben. ${ }^{12}$

\section{b) Nabestehende Unternebmen und Personen}

Der Kreis der nahestehenden Unternehmen und Personen richtet sich gem. Art. 2 lit. h ARRL II nach den Begriffsbestimmungen der im EndorsementVerfahren nach Verordnung (EG) Nr. 1606/2002 übernommenen IFRS/IAS. Eine solche Definition enthält IAS 24.9, wonach zum einen natürliche Personen einschließlich ihrer nahen Familienangehörigen erfasst werden, die die Gesellschaft beherrschen oder an ihrer gemeinschaftlichen Führung beteiligt sind, maßgeblichen Einfluss auf die Gesellschaft haben oder eine Schlüsselposition im Management der Gesellschaft oder ihrer Muttergesellschaft innehaben. Zum anderen werden auch Unternehmen einbezogen, die auf die Gesellschaft unmittelbar oder mittelbar Einfluss nehmen oder ihrem Einfluss ausgesetzt sein können. ${ }^{13}$ Den für die Konzernpraxis wichtigsten Fall aus der detaillierten Definition des Standards enthält IAS 24.9 b) i), wonach sämtliche Unternehmen derselben Unternehmensgruppe einander nahestehend sind. ${ }^{14}$

11 Dok. A8-0158/2015 vom 12.5.2015.

12 Dazu Bungert/de Raet, Der Konzern 2015, 289, $291 \mathrm{f}$.

13 Im Einzelnen dazu Senger/Prengel, in: Beck IFRS-Hdb., 5. Aufl., 2016, $\$ 20$ Rdn. 8 ff; FINK, in: Merkt/Probst/Fink, Rechnungslegung nach HGB und IFRS, 2017, Kap. 12 Rdn. 104 ff. Maßgeblicher Einfluss auf eine Gesellschaft wird nach IAS 28.5 bereits ab einem Halten von $20 \%$ der Stimmrechte widerleglich vermutet.

14 Die Unternehmensgruppe umfasst alle über Beherrschungsverhältnisse verbundenen Gesellschaften. Die Beherrschungsdefinition richtet sich nach dem control-Konzept des IFRS 10.7, vgl. EITER/SchuberT, in: Merkt/Probst/Fink, Rechnungslegung nach HGB und IFRS, 2017, Kap. $14 \mathrm{Rdn} .42 \mathrm{ff}$; zu den Unterschieden zum Konzept der $\mathbb{\int} 15 \mathrm{ff}$ AktG Windbichler, Großkomm. z. AktG, 5. Aufl., 2017, vor $\$ 15$ Rdn. 24, $\mathbb{1 7}$ Rdn. 8 . 


\section{c) Transaktion}

Bereits im Schrifttum zum Kommissionsvorschlag wurde auf das Fehlen einer Definition des Transaktionsbegriffs aufmerksam gemacht. ${ }^{15}$ Auch die nun vereinbarte Fassung der Richtlinie bestimmt den Begriff nicht. Der im Laufe des Gesetzgebungsverfahrens eingeführte Regelungsauftrag an die Mitgliedstaaten, wesentliche Transaktionen zu definieren, ${ }^{16}$ bezieht sich lediglich auf das Merkmal der Wesentlichkeit. Die genaueren Vorgaben in Art.9c Abs. 1 ARRL II zielen darauf ab, den Mitgliedstaaten die Bestimmung einer angemessenen Wesentlichkeitsschwelle zu erleichtern. Im Hinblick auf den Transaktionsbegriff geht der Unionsgesetzgeber dagegen offenbar von einem einheitlichen Transaktionsbegriff aus, der nicht im Ermessen der Mitgliedstaaten steht.

Naheliegend ist, dass sich auch der Begriff der Transaktion weitgehend nach der Definition in IAS 24.9 richtet. Erfasst wäre danach jegliche Übertragung von Ressourcen, Dienstleistungen oder Verpflichtungen zwischen der Gesellschaft und nahestehenden Unternehmen und Personen, unabhängig davon, ob ein Entgelt in Rechnung gestellt wird. Verpflichtungs- und Verfügungsgeschäfte sind gleichermaßen betroffen. Ein Blick auf den Beispielkatalog in IAS 24.11 verdeutlicht die Bandbreite einbezogener Geschäfte: Käufe- und Verkäufe, Dienstleistungen, Darlehen und Kapitaleinlagen, die Gewährung von Bürgschaften und Sicherheiten und die Eingehung von Verpflichtungen fallen sämtlich unter den Transaktionsbegriff. Für Unternehmensgruppen bedeutet dies, dass der interne Leistungs- und Lieferverkehr einschließlich CashPool-Zahlungen vollständig betroffen ist. Auch die seit Jahren im Fokus der politischen Debatte stehenden Managementbezüge fallen grundsätzlich unter den Transaktionsbegriff, ebenso Aktienausgaben im Rahmen von Kapitalerhöhungen. ${ }^{17}$

Bemerkenswert ist, dass die Mitgliedstaaten bei der Definition wesentlicher Transaktionen die Art des Geschäfts berücksichtigen dürfen. Damit dürfte auch abseits der Bereichsausnahmen nach Art. 9c Abs. 6 ARRL II etwas Spielraum für nationale Gesetzgeber bestehen, Transaktionstypen ohne Missbrauchsgefahr aus dem Anwendungsbereich der Norm zu nehmen. Dies kommt vor allem bei solchen Transaktionstypen in Betracht, deren Einbeziehung ohnehin fraglich ist. So wird die Erfassung von Gewinnausschüttungen

15 Siehe etwa Jung, WM 2014, 2351, 2353; Lanfermann/Maul, BB 2014, 1283, 1287; WiERSCH, NZG 2014, 1131, 1133.

16 Art. 9c Abs. 1 ARRL II.

17 Siehe aber die optionalen Bereichsausnahmen für Vergütungen an Mitglieder der Gesellschaftsleitung sowie für Bezugsrechtskapitalerhöhungen nach Art. 9c Abs. 6 lit. c u. e ARRL II (dazu II. 3. c) und e)). 
bereits im Schrifttum zu IAS 24 verbreitet als Versehen angesehen. ${ }^{18}$ Für die RPT-Regulierung könnten die nationalen Gesetzgeber diesbezüglich Klarheit schaffen.

Fraglich ist ebenso die Einbeziehung von Unternehmensverträgen, umwandlungsrechtlichen und sonstigen organisationsrechtlichen Maßnahmen. Obgleich die erste Fassung des IAS 24 bereits im Jahre 1984 veröffentlicht wurde, fand eine Auseinandersetzung hiermit erst im Zuge der Reform der Aktionärsrechterichtlinie statt. ${ }^{19}$ Sicherlich ist der Schutz der Gläubiger und Minderheitsaktionäre in diesen Fällen von elementarer Bedeutung. Eine Einbeziehung in die neuen RPT-Vorgaben würde jedoch vielfach zu einer unnötigen Verdopplung der nationalen Regelungen zum Außenseiterschutz führen, die im Falle von Umwandlungsmaßnahmen sogar teilweise auf EU-Richtlinien basieren. ${ }^{20}$ Auch die Beschränkung des Beispielkatalogs in IAS 24.11 auf einzelne buchungsfähige Geschäft lässt erkennen, dass das IASC (heute: IASB) tiefgreifende organisationsrechtliche Maßnahmen bei der Konzeption des Standards nicht im Blick hatte. Gleichwohl hat sich der Unionsgesetzgeber in Art. 9c ARRL II offenbar gegen eine Ausnahme organisationsrechtlicher Maßnahmen entschieden. Vielmehr ist es den Mitgliedstaaten überlassen, von der optionalen Bereichsausnahme nach Art. 9c Abs. 6 lit. b ARRL II Gebrauch zu machen, wenn nationale Regelungen für den jeweiligen Transaktionstyp das vorausgesetzte Schutzniveau erfüllen (dazu näher II. 3. b)). ${ }^{21}$

\section{d) Wesentlichkeit}

Nach Art 9c Abs. 1 ARRL II werden die Mitgliedstaaten zur Definition der Wesentlichkeit einer Transaktion aufgefordert. Zu berücksichtigen haben sie hierbei sowohl den Einfluss von Informationen über die Transaktion auf die wirtschaftlichen Entscheidungen der Aktionäre der Gesellschaft als auch die Risiken, die für die Gesellschaft und die außenstehenden Aktionäre, insbesondere Minderheitsaktionäre, mit der Transaktion verbunden sind.

Zur Bestimmung der Wesentlichkeit müssen die Mitgliedstaaten quantitative Kennzahlen festlegen oder die Art der Transaktion und die Stellung der nahe-

18 Vgl. Lüdenbach/Hoffmann/Freiberg, Haufe IFRS-Kommentar, 15. Aufl., 2017, $\$ 30$ Rdn. 33; EY, International GAAP 2017, Ch. 37, 2.7.1.

19 Vgl. J. VetTer, ZHR 179 (2015), 273, $284 \mathrm{ff}$.

$20 \mathrm{Zu}$ den drei umwandlungsrechtlichen EU-Richtlinien HABERSACK/VERSE, Europäisches Gesellschaftsrecht, 4. Aufl., 2011, \$8; LUTTER/BAYER/SCHMIDT, Europäisches Unternehmens- und Kapitalmarktrecht, 5. Aufl., 2012, \$\$ 21-23.

21 A.A. Mülbert, ZHR 179 (2015), 645, 656: Keine wesentliche Transaktion i.S.v. Art. 9c ARRL II, wenn sonstiges sekundäres Unionsrecht bereits zwingende Hauptversammlungszuständigkeit vorsehe. 
stehenden Person berücksichtigen. ${ }^{22}$ Die Kennzahlen dürfen auf dem Einfluss der Transaktion auf die Finanz-, Ertrags- und Vermögenslage, die Kapitalisierung oder den Umsatz des Unternehmens basieren. Genaue Schwellenwerte setzt die Richtlinie im Gegensatz zum Kommissionvorschlag nicht mehr. Damit wird den Mitgliedstaaten ein weites Ermessen bei der Entscheidung belassen, in welchem Maße das neue Schutzregime tatsächlich zur Anwendung kommen soll. Ausdrücklich erlaubt ist ihnen gemäß Art. 9c Abs. 1 Satz 3 ARRL II insbesondere die Festlegung verschiedener Wesentlichkeitsdefinitionen für die einzelnen Verfahrenspflichten des neuen RPT-Regimes sowie für unterschiedliche Gesellschaftsgrößen.

\section{e) Aggregation unwesentlicher Transaktionen}

Mehrere Transaktionen mit derselben nahestehenden Person werden für die Frage der Anwendbarkeit der Verfahrensvorgaben des Art. 9c ARRL II aggregiert, sofern sie innerhalb von 12 Monaten oder im selben Geschäftsjahr getätigt wurden, Art. 9c Abs. 8 ARRL II. Eine Umgehung durch Aufteilung wesentlicher Transaktionen ist somit nicht möglich. Zugleich wird sichergestellt, dass sich sukzessive verstärkende oder auf Dauer angelegte wesentliche Geschäftsverbindungen mit nahestehenden Personen nicht unkontrolliert bleiben. Der Zweck der Regelung erschöpft sich damit nicht im bloßen Schutz vor Rechtsmissbrauch. ${ }^{23}$

\section{Verfahrenspflichten}

\section{a) Öffentliche Bekanntmachung}

Art. 9c Abs. 2 ARRL II verpflichtet die Mitgliedstaaten zur Schaffung neuer Publizitätsvorschriften für RPT, die über die bisherigen Pflichten aus dem Rechnungslegungsrecht hinausgehen. Wesentliche RPT müssen danach spätestens zum Zeitpunkt des Abschlusses bekannt gegeben werden. Die Bekanntmachung muss gemäß Art. 9c Abs. 2 ARRL II mindestens Informationen über die Art des Verhältnisses zur nahestehenden Person, ihren Namen,

22 Bei der nach der deutschen Sprachfassung zwingenden Festlegung von Kennzahlen scheint es sich um einen Übersetzungsfehler zu handeln. Nach dem Kompromisstext vom 13.12.2016 (Fn. 1) sowie der gleichlautenden englischen Sprachfassung der Richtlinie ist ein Alternativverhältnis vorgesehen: „When defining material transactions Member States shall set one or more quantitative ratios [...] or take into account the nature of transaction and the position of the related party. "

23 So jedoch Bungert/de Raet, Der Konzern 2015, 289, 293; zur rechtspolitischen Bewertung der Regelung s.u. (III. 3.). 
den Zeitpunkt des Abschlusses, den Wert der Transaktion sowie alle weiteren für die Bewertung der wirtschaftlichen Angemessenheit der Transaktion erforderlichen Informationen enthalten. Da sich anhand der erstgenannten, ausdrücklich vorausgesetzten Daten regelmäßig gerade keine Bewertung der wirtschaftlichen Fairness vornehmen lässt, wird es von besonderer Bedeutung sein, inwieweit die Mitgliedstaaten genauere Informationsangaben vorgeben werden.

\section{b) Optionale Fairness Opinion}

Die Abgabe einer die Bekanntmachung flankierenden Fairness Opinion ist nur noch erforderlich, sofern die Mitgliedstaaten sie vorschreiben, Art. 9c Abs. 3 ARRL II. Im Gegensatz zum ausweislich der entsprechenden Kostenschätzung wenig durchdachten Kommissionsvorschlag einer zwingenden Bewertung durch unabhängige Dritte ${ }^{24}$ kann der Fairness-Bericht nun auch vom Verwaltungs-, Aufsichtsorgan oder einem mehrheitlich mit unabhängigen Mitgliedern besetzten Ausschuss erstellt werden. Lediglich nahestehende Personen sind bei der Vorbereitung der Stellungnahme auszuschließen. Sofern ein Mitgliedstaat die Veröffentlichung einer Fairness Opinion vorschreibt, muss diese auch Erläuterungen der zugrunde gelegten Annahmen und angewandten Methoden enthalten.

Aussagen zur Marktüblichkeit der Transaktion werden im verabschiedeten Richtlinientext nicht mehr ausdrücklich vorgeschrieben. ${ }^{25}$ Wo seine Anwendung möglich ist, dürfte jedoch weiterhin der international anerkannte Fremdvergleichsmaßstab (arm's length principle) zur Beurteilung der Angemessenheit anzulegen sein. $\mathrm{Ob}$ ein Geschäft darüber hinaus auch „vernünftig“ im Sinne von Art. 9c Abs. 3 ARRL II ist, wird sich jenseits evidenter Missbrauchsfälle dagegen kaum beurteilen lassen. Der Voraussetzung kommt eine lediglich geringe eigenständige Bedeutung zu.

\section{c) Zustimmungsvorbehalt und Stimmverbot}

Für ihren Vorschlag, die Hauptversammlung zwingend über wesentliche RPT abstimmen zu lassen, erntete die Kommission vorwiegend aus Deutschland massive Kritik. Die zahlreichen Gründe, die eine solche Zuständigkeit zum systemfremden Störelement im deutschen Recht gemacht hätten, wurden im

24 Ausgegangen war die Kommission von Gutachterkosten in Höhe von maximal 2500 bis 5000 EUR, siehe S. 10 des Vorschlags (Fn. 4); zur Abwegigkeit dieser Kostenschätzung FleisCher, BB 2014, 2691, 2698; J. VetTER, ZHR 179 (2015), 273, 311.

25 So noch Art. 9c Abs. 1 Satz 1 im Vorschlag der Kommission (Fn. 4). 
Schrifttum ausführlich dargelegt und ließen auch die EU-Institutionen alsbald von dem Vorhaben abrücken. ${ }^{26}$ Für wesentliche RPT müssen die Mitgliedstaaten nun gemäß Art. 9c Abs. 4 ARRL II einen Zustimmungsvorbehalt der Hauptversammlung oder des Verwaltungs- oder Aufsichtsorgans der Gesellschaft vorschreiben. Optional kann auch eine zusätzliche Hauptversammlungsabstimmung nach erfolgter Zustimmung des Verwaltungs- oder Aufsichtsorgans vorgesehen werden.

Fragen wirft selbst nach vielen Überarbeitungen im Gesetzgebungsverfahren das in der Abstimmung gemäß Art. 9c Abs. 4 Satz 3 ARRL II vorgesehene Stimmverbot der nahestehenden Person auf. So dürfen an der Transaktion beteiligte Aktionäre, Verwaltungs- und Aufsichtsratsmitglieder grundsätzlich nicht am Abstimmungsverfahren teilnehmen. Das Abstimmungsverfahren muss außerdem dergestalt konzipiert sein, dass die nahestehende Person ihre Stellung nicht ausnutzen kann und die Gesellschaft sowie außenstehende Aktionäre angemessen geschützt werden. Inwieweit daraus auch Beschränkungen für Abhängige und Vertreter einer nahestehenden Person folgen, wird nicht ausdrücklich dargelegt. ${ }^{27}$ Insbesondere findet sich im endgültigen Richtlinientext das in Art. 9c Abs. 2 a des Parlamentsentwurfs ${ }^{28}$ vorgesehene ausdrückliche Stimmverbot für Vertreter von nahestehenden Personen nicht mehr.

Die weiteren Ausführungen des Art. 9c Abs. 4 ARRL II lassen jedoch erkennen, welches Schutzniveau der europäische Gesetzgeber erwartet: So dürfen die Mitgliedstaaten eine Teilnahme nahestehender Paketaktionäre an der Abstimmung gemäß Art. 9c Abs. 4 Satz 4 ARRL II nur vorsehen, wenn sie diese in der Hauptversammlung oder im Verwaltungs- oder Aufsichtsorgan nicht gegen die Mehrheit der außenstehenden Aktionäre (bei Abstimmung in der Hauptversammlung) beziehungsweise unabhängigen Organmitglieder (bei Abstimmung im Verwaltungs- oder Aufsichtsorgan) durchsetzen können. Ausdrücklich wird zudem klargestellt, dass ein solcher Schutz vor oder während der Abstimmung greifen muss. Beispielhaft schlägt Erwägungsgrund 43 der Änderungsrichtlinie qualifizierte Mehrheitserfordernisse vor.

Damit steht zum einen fest, dass selbst umfangreiche nachträgliche Berichts-, Prüfungs- und Ausgleichspflichten, wie sie etwa das Recht des faktischen

26 Dazu Bayer/Selentin, NZG 2015, 7, 8; Bremer, NZG 2014, 425; Drygala, AG 2013, 198, 208 ff; Fleischer, BB 2014, 2691, 2698 ff; Hommelhoff, KSzW 2014, 63, 67; Jung, WM 2014, 2351, 2354; Lanfermann/Maul, BB 2014, 1283, 1287 f; U. H. Schneider, EuZW 2014, 641, 642; SeIBT, DB 2014, 1910, 1914 f; Verse/Wiersch, EuZW 2014, 375, 380; VetTer, ZHR 179 (2015), 273, 304 ff; Wiersch, NZG 2014, 1131, 1136; ZEtZsche, NZG 2014, 1121, $1126 \mathrm{f}$.

27 Für eine restriktive Auslegung Spindler/SEIdel, AG 2017, 169, $172 \mathrm{f}$.

28 Dok. 10626/15 vom 22.7.2015. 
Konzerns in Deutschland vorsieht, als stimmverbotskompensierende Schutzmechanismen nicht genügen. Zum anderen muss bei zweckgemäßer Auslegung des Art. 9c Abs. 4 ARRL II davon ausgegangen werden, dass abhängige Verwaltungs- oder Aufsichtsorganmitglieder auch bei Ausschluss der nahestehenden Person vom Abstimmungsverfahren nicht zur Überstimmung der Unabhängigen in der Lage sein dürfen. In dieser Hinsicht schafft der europäische Gesetzgeber in der seit Jahren anhaltenden Kontroverse um die Besetzung von Verwaltungsorganen mit unabhängigen Mitgliedern ${ }^{29}$ Fakten: Ohne die Unabhängigen wird eine Übertragung der erforderlichen Zustimmungskompetenz auf diese Organe nicht möglich sein.

\section{d) Erleichterungen für marktgerechte Transaktionen im ordentlichen Geschäftsgang}

Marktübliche Transaktionen im ordentlichen Geschäftsgang sind von den Verfahrensregelungen des Art. 9c ARRL II ausgenommen, sofern die Mitgliedstaaten ihre Einbeziehung nicht ausdrücklich vorsehen. Stattdessen muss das Verwaltungs- oder Aufsichtsorgan der Gesellschaft ein internes Verfahren einrichten, in dem periodisch überprüft wird, dass stattgefundene Transaktionen im ordentlichen Geschäftsgang und zu marktüblichen Konditionen erfolgt sind. Transaktionen, die die Bedingungen erfüllen, unterliegen somit einem eigenen Kontrollverfahren und fallen auch nicht unter die Aggregationsregelung des Art. 9c Abs. 8 ARRL II. Damit ist der laufende Leistungs- und Lieferverkehr zwischen Konzerngesellschaften von den aufwändigen Verfahrenspflichten der Art. 9c Abs. 2 bis 4 ARRL II befreit. Dies gilt insbesondere für die täglichen Zahlungsströme im konzerninternen Cash-Pooling, nicht jedoch für die zugrunde liegende Rahmenvereinbarung. ${ }^{30}$

\section{e) Öffentliche Bekanntmachung von Transaktionen der Tochtergesellschaften}

Wesentliche Transaktionen zwischen nahestehenden Unternehmen oder Personen einer Gesellschaft und ihren Tochtergesellschaften müssen nach Art. 9c Abs. 7 ARRL II ebenfalls öffentlich bekannt gemacht werden. Die Voraussetzung einer Fairness Opinion liegt erneut im Ermessen der Mitgliedstaaten.

29 Dazu Baums, ZHR 180 (2016), 697; Bayer, NZG 2013, 1, 10 ff; Hommelhoff, ZIP 2013, 953; DERs., ZIP 2013, 1645; Hopt, ZHR 175 (2011), 444, 472ff; Rотн, ZHR 175 (2011), 605; aus dem internationalen Schrifttum Gilson/Kraakman, Stan. L. Rev. 43 (1991), 863; Gordon, Stan. L. Rev. 59 (2007), 1465; Gutiérrez/SÁez, J. Corp. L. Stud. 13 (2013), 63; Ringe, EBOR 2013, 401.

$30 \mathrm{Zu}$ Funktion und Ausgestaltung von Rahmenvereinbarungen für den konzerninternen Leistungsverkehr HommeLHoff, ZGR 2012, 535, $542 \mathrm{ff}$. 
Die Regelung soll eine Umgehung der Verfahrensvorschriften unter Ausnutzung von Tochtergesellschaften verhindern. ${ }^{31}$ Maßgeblich für die Bestimmung der Wesentlichkeitsschwelle sind daher nicht die Kennzahlen der Tochter-, sondern die der Muttergesellschaft. Das entspricht dem Normzweck, der sich auf den Schutz der Muttergesellschaft und ihrer außenstehenden Aktionäre begrenzt, und verhindert eine übermäßige Ausdehnung der Regelungen auf nicht börsennotierte Tochtergesellschaften. Vor diesem Hintergrund ist auch das Fehlen eines Zustimmungserfordernisses zu sehen, wie es Art. 9c Abs. 4 ARRL II für direkt mit der Muttergesellschaft abgeschlossene RPT vorsieht. Mit der rechtlichen Selbstständigkeit der Tochtergesellschaft wäre es schwer vereinbar, ihre Geschäfte von der Zustimmung von Organen der Muttergesellschaft abhängig zu machen.

\section{Bereichsausnabmen}

Art. 9c Abs. 6 ARRL II enthält einen Katalog von Transaktionstypen, die nach dem Willen der Mitgliedstaaten von den Verfahrenspflichten befreit oder deren Befreiung in das Ermessen der betroffenen Gesellschaft gestellt werden kann.

\section{a) Transaktionen mit Tochtergesellschaften}

Transaktionen mit Tochtergesellschaften können nach Art. 9c Abs. 6 lit. a ARRL II befreit werden, wenn die Gesellschaft sämtliche Anteile der Tochtergesellschaft oder keine andere nahestehende Person Anteile an ihr hält. Eine Befreiung ist außerdem möglich, wenn das nationale Recht einen angemessenen Alternativschutz für die Gesellschaft, die Tochtergesellschaft sowie die außenstehenden Aktionäre beider Gesellschaften vorsieht. Die Regelung erlaubt die Ausnahme einer Vielzahl von Transaktionen, für die schon keine Gefährdung der Interessen außenstehender Aktionäre besteht oder der bezweckte Schutz ihrer Interessen anderweitig gewährleistet ist. Warum der europäische Gesetzgeber es an dieser Stelle für notwendig erachtet, auch den Schutz der Tochtergesellschaft und ihrer außenstehenden Minderheitsaktionäre vorzusehen, bleibt schleierhaft. ${ }^{32}$ Die Verfahrenspflichten des Art. 9c ARRL II sehen gerade keinen umfassenden Schutz aller Gruppengesellschaften im Konzern vor, sondern beschränken sich auf die Interessenwahrung derjenigen Gesellschaft, die diesen Pflichten unterliegt.

31 Zur diesbezüglichen Lücke im Kommissionsentwurf ENRIQUEs, EBOR 2015, 1, 28; TrÖgER, AG 2015, 53, $62 \mathrm{f}$.

32 Kritisch bereits Tröger, AG 2015, 53, 64. 


\section{b) Obnebin zustimmungsbedürftige Transaktionen}

Klar definierte Transaktionsarten, für die das jeweilige nationale Recht ohnehin eine Abstimmung in der Hauptversammlung vorschreibt, können nach Art. 9c Abs. 6 lit. b ARRL II ebenfalls ausgenommen werden. Angaben zur Ausgestaltung der Abstimmung und insbesondere zu etwaigen Stimmverboten macht die Richtlinie nicht. Allerdings müssen die faire Behandlung aller Aktionäre sowie der Schutz der Interessen der Gesellschaft und der außenstehenden Aktionäre durch spezifische Regelung für den jeweiligen Transaktionstyp angemessen sichergestellt sein. Die optionale Bereichsausnahme gilt nicht für Transaktionen, die nach nationalem Recht einem Zustimmungsvorbehalt des Verwaltungs- oder Aufsichtsorgans unterliegen. Lediglich bei Einbeziehung der Hauptversammlung lässt die Richtlinie den Mitgliedstaaten mehr Raum für flexiblere Regelungen des Aktionärsschutzes. Offenbar teilt der europäische Gesetzgeber die international herrschende Auffassung, ein optimaler Schutz vor missbräuchlichen RPT sei letztlich nur unter Mitwirkung der Hauptversammlung zu erreichen. ${ }^{33}$

\section{c) Vergütung von Mitgliedern der Unternehmensleitung}

Vergütungsleistungen an Mitglieder der Unternehmensleitung können gemäß Art. 9 c Abs. 6 lit. c ARRL II ausgenommen werden, wenn sie im Einklang mit den Bestimmungen des neuen Art. 9a ARRL II gewährt werden. Dieser regelt bereits den Schutz der Aktionäre vor übermäßigen Managementvergütungen, indem er Gesellschaften die Pflicht zur Erarbeitung einer Vergütungspolitik sowie die Genehmigung derselben durch die Hauptversammlung vorsieht. ${ }^{34}$

\section{d) Stabilisierungsmaßnabmen für Kreditinstitute}

Für Transaktionen von Kreditinstituten, die auf der Basis von Maßnahmen der Aufsichtsbehörden zur Stabilitätssicherung im Sinne des Unionsrechts durchgeführt werden, ist nach Art. 9c Abs. 6 lit. d ARRL II ebenfalls eine optionale Bereichsausnahme vorgesehen.

33 Vgl. Djankov/La Porta/Lopez-de-Silanes/Shleifer, Journ. Financ. Econ. 88 (2008), 430, 463; Enriques, EBOR 2015, 1, 15; Hamdani/Yafeh, Review of Finance 17 (2013), 691, 692.

34 Die Mitgliedstaaten können der entsprechenden Abstimmung in der Hauptversammlung jedoch lediglich empfehlenden Charakter zusprechen, Art. 9a Abs. 3 ARRL II. Die Möglichkeit nach Art. 9c Abs. 6 lit. c ARRL II, Vergütungsleistungen an Mitglieder der Unternehmensleitung von den RPT-Verfahrenspflichten auszunehmen, bleibt hiervon unberührt. 


\section{e) Angebote an alle Aktionäre}

Schließlich können Transaktionen ausgenommen werden, die allen Aktionären zu gleichen Konditionen angeboten werden, wenn die Gleichbehandlung der Aktionäre und der Schutz der Gesellschaftsinteressen gewahrt sind, Art. 9c Abs. 6 lit. e ARRL II. Die wichtigsten dieser Geschäfte, namentlich Dividendenzahlungen und Bezugsrechtsemissionen, ${ }^{35}$ fallen freilich schon unter die Ausnahmeoption nach Art. 9c Abs. 6 lit. b ARRL II.

\section{Sanktionen}

Gemäß Art. 14b ARRL II müssen die Mitgliedstaaten für Verstöße gegen die aufgrund der Richtlinie erlassenen innerstaatlichen Vorschriften Maßnahmen und Sanktionen festlegen und auch die zu ihrer Anwendung erforderlichen Maßnahmen treffen. Anhaltspunkte für eine zwingende Unwirksamkeit von Transaktionen bei Verstößen gegen die neuen Regelungen finden sich im verabschiedeten Richtlinientext nicht mehr. ${ }^{36}$ Die Auswahl der erforderlichen Sanktionsmittel liegt somit ganz im Ermessen des jeweiligen nationalen Gesetzgebers. Lediglich deren Effektivität, Angemessenheit und abschreckende Wirkung ist zu gewährleisten.

\section{Umsetzungsperspektiven in Deutschland}

Während sich der Kommissionsvorschlag der neuen Richtlinie noch augenfällig die UK Listing Rules zum Vorbild genommen hatte, ${ }^{37}$ wurde die Nähe zum britischen Modell im Folgenden reduziert. Die zahlreichen Änderungen und Ergänzungen tragen vornehmlich die Handschrift der italienischen Ratspräsidentschaft in der zweiten Jahreshälfte 2014, deren letzte Kompromissvorschläge bereits weitgehend konsensfähig waren. ${ }^{38}$ Entsprechend finden sich

35 Eine ähnliche Ausnahmeregelung im Kompromissvorschlag der italienischen Ratspräsidentschaft vom 10.11.2014 führte beide Transaktionstypen noch explizit als Beispiele auf, siehe Dok. Nr. 13758/14, Art. 9 c Abs. 4 lit. c.

36 Zur Unwirksamkeit als naheliegende Verstoßfolge nach dem Kommissionsvorschlag der Richtlinie DAV-Handelsrechtsausschuss, NZG 2015, 54, 65; Seibt, DB 2014, 1910, 1914; J. VETTER, ZHR 179 (2015), 273, 288 f.

37 Siehe FCA Handbook LR 11.1; dazu Drygala, AG 2013, 198, 209; BöckLI, SZW 2016, 444, 458f, sieht den Ursprung der vorgeschlagenen Regelungen auch im französischen Aktienrecht.

38 Vgl. Fleischer, ZGR 2017, 1, 11; siehe auch die ausführliche Analyse der italienischen Kompromissvorschläge bei Jung, WM 2014, 2351 (Vorschläge 1 und 2); J. VETTER, ZHR 179 (2015), 273, $279 \mathrm{ff}$ (Vorschläge 1 bis 3). 
im verabschiedeten Richtlinientext Impulse aus den italienischen RPT-Regelungen der Kapitalmarktaufsicht $\mathrm{CONSOB}^{39}$, die ihrerseits eine moderne kontinentaleuropäische Antwort auf die angloamerikanischen Regelungsmodelle für RPT sind. ${ }^{40}$ Große Weichenstellungen auf dem Weg zu einer für kontinentaleuropäische Mitgliedstaaten tragfähigen Regelung waren insbesondere die wahlweise Kompetenzübertragung für die Fairness Opinion und das Zustimmungserfordernis auf das Verwaltungs- oder Aufsichtsorgan.

Trotz gewachsenen Umfangs reicht die verabschiedete Fassung von Art. 9c ARRL II in ihrem Detailgrad nicht an die entsprechenden Regelungen der britischen und italienischen Vorbilder heran. Dies ist einerseits zu begrüßen, bleibt den Mitgliedstaaten dadurch doch Flexibilität bei der Anpassung des EU-Regimes an die jeweiligen Rechtssysteme und Märkte. Andererseits können die vorgesehenen Verfahrenspflichten den ausgeprägten Liefer- und Leistungsverkehr innerhalb von Unternehmensgruppen je nach ihrer konkreten Ausgestaltung massiv beeinträchtigen. Minimalistische Umsetzungsinitiativen werden daher nicht genügen - vielmehr ist der deutsche Gesetzgeber nun in der Pflicht zur ausgefeilten Umsetzung, will er seinen börsennotierten Gesellschaften keine unnötigen Hürden im globalen Wettbewerb aufbürden. Wertvolle Anstöße können hierbei die britischen und italienischen RPT-Regelungen liefern. Im Einzelnen:

\section{Notwendigkeit einer vollständigen RPT-Definition}

Die detaillierte Definition der „related parties“ in IAS 24.9 wurde im Laufe der Jahre mehrfach präzisiert. Abgrenzungsschwierigkeiten beschränken sich im Wesentlichen nur noch auf die Frage, inwieweit neben Kindern und Ehegatten von nahestehenden Personen auch entferntere Familienangehörige einzubeziehen sind. ${ }^{41} \mathrm{Um}$ auch im Umgang mit dem Transaktionsbegriff keine Unsicherheiten aufkommen zu lassen, wäre für die Umsetzung der Richtlinie eine Übernahme der entsprechenden Definition in IAS 24.9 zu empfehlen.

Wenngleich der Rückgriff auf die Begriffsbestimmungen der IAS vor dem Hintergrund ihrer europaweiten Vertrautheit zweckdienlich ist, bleibt zu bedenken, dass die Begriffsweite dieser Definitionen der geringen Eingriffs-

39 CONSOB, Regolamento operazioni con parti correlate, Delibera n. 17221 vom 12.3.2010.

40 Zur Vorbildfunktion der angloamerikanischen Regelungsmodelle bei der Konzeption der italienischen Regelungen siehe CONSOB, Consultation document on the regulation of related party transactions, 9.4.2008, Executive Summary S. $1 \mathrm{f}$, abrufbar unter http:// wrw.consob.it/documents/46180/46181/cons_em_20080409_exec_sum.pdf/43888058Oaf9-4a62-a427-2c5a2865fb78.

41 Vgl. Senger/Prengel, aaO (Fn. 13), $\$ 20$ Rdn 25 ff; Lüdenbach/Hoffmann/Freiberg, aaO (Fn. 18), $\$ 30$ Rdn. 18. 
intensität der IFRS/IAS entspricht. Für die über bloße Publizitätsanforderungen hinausgehenden Pflichten in Art. 9c ARRL II sind sie gewiss zu weit. ${ }^{42}$ Eine Begrenzung des Anwendungsbereich über hinreichend hohe Wesentlichkeitsschwellen sowie eine Ausschöpfung des Ausnahmekatalogs nach Art. 9c Abs. 6 ARRL II im Zuge der Richtlinienumsetzung ist daher unerlässlich.

\section{Schlüsselfunktion der Wesentlichkeitsschwelle}

Die wichtigste Stellschraube in den europäischen RPT-Vorgaben ist die von den Mitgliedstaaten aufzustellende Wesentlichkeitsdefinition. Rechtspolitisch ist die diesbezügliche Zurückhaltung der EU-Institutionen zu beklagen: Im Kontrast zur Ausgestaltung von Schutznormen, bei der die rechtlichen und ökonomischen Eigenheiten der Mitgliedstaaten berücksichtigt werden müssen, eignet sich die Frage des Anwendungsbereichs hervorragend zur Harmonisierung. ${ }^{43}$ Die von der Kommission vorgesehenen Schwellenwerte von $1 \%$ und $5 \%$ des Gesellschaftsvermögens wurden dennoch schon im ersten Kompromissvorschlag der italienischen Ratspräsidentschaft ${ }^{44}$ aufgegeben.

Für das deutsche Recht schlagen Bungert und de Raet vor, dass die festzulegenden Schwellenwerte nicht die Wertgrenzen der nach $\$ 111$ Abs. 4 Satz 2 AktG zustimmungsvorbehaltsfähigen Geschäften unterschreiten sollten. ${ }^{45} \mathrm{Im}$ Hinblick auf die Zustimmungserfordernisse der Richtlinie ist dem zuzustimmen. Niedrigere Schwellenwerte würden ohne Not in die Leitungsautonomie des Vorstands eingreifen. Im Gegensatz zu $\ 111$ Abs. 4 Satz 2 AktG sollte sich der Gesetzgeber bei der Umsetzung der neuen Richtlinie auch nicht der Nennung konkreter quantitativer Wertgrenzen entziehen. Zwar lässt die Richtlinie eine Bestimmung anhand ausschließlich qualitativer Kriterien und damit eine auf den Einzelfall angepasste Wesentlichkeitsbeurteilung zu. ${ }^{46} \mathrm{Um}$ einerseits eine rechtssichere Handhabung zu ermöglichen und andererseits nicht der im Interessenkonflikt stehenden Geschäftsführung die Beurteilung des Eingreifens der neuen Regelungen zu überlassen, ist die Hinzuziehung

42 Dazu Bungert/de Raet, Der Konzern 2015, 289, 292; Bayer/Selentin, NZG 2015, 7, 10; kritisch insbesondere LUTTER, EuZW 2014, $687 \mathrm{f}$.

43 J. Vetter, ZHR 179 (2015), 273, 318; a. A. Bayer/Selentin, NZG 2015, 7, 9; Bungert/ DE RAET, Der Konzern 2015, 289, 293.

44 Dok. 13758/14 vom 10.11.2014.

45 BungerT/De RaET, Der Konzern 2015, 289, 293; als Untergrenze zustimmungsvorbehaltsfähiger Geschäfte wird im Schrifttum verbreitet $1 \%$ einer geeigneten Bezugsgröße genannt, vgl. FleisCHER, BB 2013, 835, 842.

46 Zur in diesem Punkt engeren deutschen Sprachfassung der Richtlinie s. o. (Fn. 22). 
quantitativer Kriterien jedoch dringend zu empfehlen. ${ }^{47}$ Nicht zuletzt wäre auch die Anwendung der Aggregationsregelung nach Art. 9c Abs. 8 ARRL II ohne quantitative Wertgrenzen kaum möglich.

Als passable Schwelle für den Zustimmungsvorbehalt erscheinen aus rechtsvergleichender Perspektive die bereits von der Kommission vorgeschlagenen $5 \%{ }^{48}$ Sowohl das britische als auch das italienische RPT-Regime greifen erst bei Erreichen ${ }^{49}$ bzw. Überschreiten ${ }^{50}$ dieses Werts vollumfänglich ein. Neben der ursprünglich vorgesehenen Bezugsgröße „Vermögen“"51 (gemeint war vermutlich das bilanzielle Aktivvermögen $)^{52}$ sollten entsprechend der britischen ${ }^{53}$ und italienischen ${ }^{54}$ Regelungen auch weitere Bezugsgrößen wie Eigenkapital und Gewinn als alternative Schwellen herangezogen werden, um eine möglichst feinmaschige Erfassung wesentlicher Transaktionen sicherzustellen. Empfehlenswert ist ferner eine klare Regelung zur Bewertung von Dauerschuldverhältnissen..$^{55}$

Für die Publizitätsanforderungen in Art. 9c Abs. 2, 3 ARRL II sind auch Wertgrenzen unter der Schwelle des $\$ 111$ Abs. 4 Satz 2 AktG möglich, nach hier vertretener Ansicht jedoch nicht notwendig. Im praktisch wichtigsten Fall der related party transactions, dem Leistungsaustausch im faktischen Konzern, ist über den Abhängigkeitsbericht nach $\$ 312$ AktG bereits unabhängig von Wertgrenzen eine Überprüfung sämtlicher Geschäfte sichergestellt. Will der deutsche Gesetzgeber hier für mehr Transparenz sorgen, so erscheint es geeigneter, die umstrittene Vertraulichkeit des Abhängigkeitsberichts zumindest in Teilen aufzuheben. ${ }^{56}$

47 So auch Enriques, EBOR 2015, 1, $27 \mathrm{f}$.

48 So auch J. VetTer, ZHR 179 (2015), 273, 319; a.A. noch zum Kommissionsvorschlag Lanfermann/Maul, BB 2014, 1283, 1287.

49 FCA Handbook LR 11.1.10.

50 CONSOB, aaO (Fn. 39), Annex 3, Abs. 1.1.

51 Art. 9c Abs. 1 und 2 des Kommissionsvorschlags (Fn. 4).

52 Lanfermann/Maul, BB 2014, 1283, 1287; Seibt, DB 2014, 1910, 1914 Fn. 44.

53 FCA Handbook LR 10 Annex 1; zu diesen sog. class tests Pearce/Kirkby, in: Blair/ Walker/Willey, Financial Markets and Exchanges Law, 2. Aufl., 2012, S. 173, 183 f; zu den aktuellen Reformüberlegungen FCA Consultation Paper CP17/4, Februar 2017, Rdn. 4.1ff, abrufbar unter https://www.fca.org.uk/publication/consultation/cp17-04. $p d f$.

54 COnsob, aaO (Fn. 39), Annex 3, Abs. 1.1 lit. a-c.

55 Vgl. SеibT, BDI/FBD: Erforderliche Harmonisierung oder unnötiger Systembruch Der Vorschlag der EU-Kommission zu Related Party Transactions, 2014, S. 11.

56 Dazu Fleischer, BB 2014, 835; J. Vetter, in: Fleischer/Koch/Kropf/Lutter, 50 Jahre AktG, 2015, S. 231, 253 ff; stattdessen für eine erweiterte Berichterstattung im Lagebericht Hommelhoff, Gutachten G für den 59. DJT, 1992, G 57 ff. 
Im Übrigen könnte der deutsche Gesetzgeber eine Privilegierung von KMU und Wachstumsunternehmen erwägen, um diesen den Zugang zum regulierten Markt nicht weiter zu erschweren. ${ }^{57}$ Eine ausdrückliche Ausnahme sieht Art. 9c Abs. 1 ARRL II diesbezüglich zwar nicht vor, wohl aber die Möglichkeit der Differenzierung der Wesentlichkeitsschwelle nach Unternehmensgrößen.

\section{RPT-Aggregation als Bürokratiemonster im Konzern}

Die Aggregationsregelung für Transaktionen mit derselben nahestehenden Person hat im Schrifttum zu Recht für Irritationen gesorgt: Wie sollen die Vorgaben nach Art. 9c Abs. 2 bis 4 ARRL II noch erfüllt werden, wenn eine Transaktion erst im Rahmen der Aggregation nachträglich die Wesentlichkeitskriterien erfülltt? ${ }^{58}$ Eine „spätestens zum Zeitpunkt ihres Abschlusses“59 erfolgende Bekanntmachung und Fairness Opinion ist dann nicht mehr möglich. Auch eine erst Monate später eintretende Zustimmungspflicht mitsamt der einhergehenden Rechtsunsicherheit kann der Unionsgesetzgeber nicht ernstlich gewollt haben. Vielmehr ging die Klarheit der Regelung im Zuge von Umformulierungen und Anpassungen an den restlichen Normtext während der italienischen Ratspräsidentschaft verloren. ${ }^{60}$ Richtigerweise kann erst diejenige Transaktion, bei deren Addierung mit vorangegangenen Transaktionen der letzten 12 Monate die Wesentlichkeitsschwelle überschritten wird, zustimmungspflichtig sein und muss spätestens im Zeitpunkt ihres Abschlusses, gegebenenfalls mit einer Fairness Opinion, bekannt gemacht werden. Über vorangegangene Geschäfte, die aggregiert wurden, muss erst im Rahmen dieser Bekanntmachung, je nach nationalem Recht ebenfalls mit einer begleitenden Fairness Opinion, berichtet werden. Die Publizitäts- und Zustimmungspflichten gelten sodann für jede einzelne nachfolgende Transaktion, solange die Aggregation mit den Transaktionen der vergangenen 12 Monate zur Erfüllung des Wesentlichkeitskriteriums führt.

Die Unklarheiten im Umgang mit der Aggregationsvorschrift kann der deutsche Gesetzgeber durch präzise Regelung nach dem Vorbild der Listing Rules ${ }^{61}$ beseitigen. Der immense bürokratische Aufwand, der durch die Regelung ent-

57 Vgl. dazu Veil, Kapitalmarktzugang für Wachstumsunternehmen, 2016, S. 150, 160.

58 Vgl. Bungert/De Raet, Der Konzern 2015, 289, 293.

59 Art. 9c Abs. 2 ARRL II.

60 Art. 9c Abs. 3 ARRL II des Kommissionsvorschlags (Fn. 4) war diesbezüglich noch klarer, bezog sich jedoch nur auf die Zustimmungspflicht; dazu (mit erläuterndem Beispiel) ZeTZsche, NZG 2014, 1121, 1126.

61 Siehe FCA Handbook LR 11.1.11 sowie UKLA Technical Note 307.1. 
steht, ist dagegen kaum vermeidbar. ${ }^{62}$ In integrierten Konzernen dürfte es schlicht nicht zu bewerkstelligen sein, jedes einzelne Geschäft mit derselben Konzerngesellschaft zu veröffentlichen und zu genehmigen, sobald die vorigen Transaktionen zusammengenommen die Wesentlichkeitsschwelle überschritten haben. Der Verzicht auf eine Fairness Opinion, die Ausschöpfung der optionalen Bereichsausnahmen sowie eine extensive Auslegung der Ausnahme von Transaktionen im ordentlichen Geschäftsgang können das Problem nur einschränken. Aufgrund der Aggregationsregelung wird es nicht reichen, ausschließlich wesentliche Geschäfte mit nahestehenden Personen zu identifizieren. Börsennotierte Gesellschaften werden vielmehr ein internes Überwachungssystem für sämtliche RPT einrichten müssen, um das Überschreiten der Wesentlichkeit bei aggregierten Transaktionen rechtzeitig erkennen zu können. Ein Blick auf das Vereinigte Königreich veranschaulicht, dass der Regelungstyp keinerlei Fahrlässigkeit zulässt:

Exillon Energy plc führte nach ihrem Börsengang im Jahr 2009 die bestehende Praxis fort, private Ausgaben ihres damaligen chairman zunächst zu bezahlen und später mit der noch unbezahlten Vergütung zu verrechnen. Eine dadurch entstandene Schädigung der Gesellschaft stellte die zuständige Aufsichtsbehörde FSA (heute: FCA) nicht fest. Da die Zahlungen jedoch innerhalb von 6 Monaten zusammengerechnet die Schwelle der Offenlegungspflichten für kleinere RPT nach LR 11.1.10 überschritten, wertete die FSA sie als Darlehen an eine related party. Gegen die Gesellschaft wurde wegen Verstoßes gegen die Bekanntmachungspflichten in LR 11.1.10 sowie gegen die Pflicht zur Einrichtung geeigneter Compliance-Mechanismen aus Listing Principle 2 eine Strafzahlung von $£ 292950$ verhängt. ${ }^{63}$

\section{Sinnvolle Ergänzung bestehender Publizitätspflichten}

Eine verbindliche Abwägung des Publizitätsinteresses der Aktionäre mit den Geheimhaltungsinteressen der Gesellschaft wollte der europäische Gesetzgeber offenbar für die Bekanntmachungspflichten nicht vornehmen. Die Aufgabe, konkrete Vorgaben hinsichtlich der zu veröffentlichenden Informationen nach Art. 9c Abs. 2 ARRL II zu machen, liegt nun in den Händen des deutschen Gesetzgebers. Fest steht, dass auch bei einer Vergabe der Zustimmungskompetenz auf den Aufsichtsrat kein geringerer Informationsbedarf der Aktionäre besteht. Haben die Aktionäre auch kein Vetorecht, so bleibt ihnen bei ausreichender Informationslage doch die frühzeitige Abstimmung mit den

62 Kritisch zur RPT-Aggregation auch Bungert/de Raet, Der Konzern 2015, 289, 293; J. Vetter, ZHR 179 (2015), 273, 322; Wiersch, NZG 2014, 1131, 1132.

63 FSA Final Notice vom 26.4.2012, abrufbar unter https://www.fca.org.uk/publication/ final-notices/exillon-energy.pdf. 
Füßen. ${ }^{64}$ Alle für die Bewertung der Fairness und Vernünftigkeit der Transaktion notwendigen Informationen sind daher zu veröffentlichen. ${ }^{65}$

Neben den ausdrücklich in Art. 9c Abs. 2 ARRL II genannten Vorgaben dürfte entsprechend der britischen Regelungen für kleinere RPT zumindest eine Beschreibung des Geschäfts notwendig sein. ${ }^{66}$ Welche Informationen darüber hinaus als bewertungsrelevant erachtet werden können, demonstrieren die detaillierten Veröffentlichungskataloge in den britischen und italienischen Regelungen für größere RPT. ${ }^{67}$ Die Erweiterung der Publizitätspflichten durch die neue Richtlinie wurde auch in Deutschland weitgehend begrüßt. ${ }^{68}$ In der Tat ergänzt sie die bestehenden Publizitätspflichten auf sinnvolle Weise: Bleibt der Abhängigkeitsbericht auch in Zukunft vertraulich, so werden außenstehende Aktionäre unabhängig vom Bilanzierungssystem der Gesellschaft zumindest über die wichtigsten RPT spätestens zum Zeitpunkt des Abschlusses informiert werden.

\section{Feblende Notwendigkeit einer Fairness Opinion}

Die Ausgestaltung der nach Art. 9c Abs. 3 ARRL II möglichen Vorgabe einer Fairness Opinion stünde dem deutschen Gesetzgeber weitgehend frei. Für das deutsche Recht stellt sich angesichts der Prüfung des Abhängigkeitsberichts nach $₫ 313$ AktG die Frage, ob eine zusätzliche unabhängige Bewertung erforderlich ist. Im Vergleich zu bloßen Veröffentlichungspflichten ist die Einholung einer unabhängigen Fairness Opinion mit deutlich höheren Kosten verbunden. Vergibt der deutsche Gesetzgeber die Zustimmungskompetenz für RPT an den Aufsichtsrat, erschöpft sich der Nutzen in der früheren Information der Aktionäre. ${ }^{69}$ Kosten und Nutzen einer unabhängigen Bewertung stünden in keinem angemessenen Verhältnis zueinander.

64 Grundlegend zur Bedeutung beider Rechte Hirschman, Exit, voice and loyalty, 1970, passim.

65 Vor einer Offenlegung sämtlicher bewertungsrelevanter Informationen warnend DAVHandelsRechtsausschuss, NZG 2015, 54, 64 („nicht praktikabel“).

66 FCA Handbook LR 11.1.10 Abs. 2 lit. c.

67 Siehe FCA Handbook LR 11.1.7 i.V.m. LR 10.4.1, 13.3 und 13.6 bzw. СоNsOB, aaO (Fn. 39), Annex 4.

68 Vgl. Bayer/Selentin, NZG 2015, 7, 13; Drygala, AG 2013, 198, 207 f; Fleischer, BB 2014, 2691, 2698; Hommelhoff, KSzW 2014, 63, 66f; U. H. Schneider, EuZW 2014, 641, 642; J. VetTer, ZHR 179 (2015), 273, 313 f; jüngst J. Schmidt, Der Konzern 2017, 1, 10; kritisch DAV-Handelsrechtsausschuss, NZG 2015, 54, 62f; Simon, DB 4/2015, M5.

69 Kritisch zur Aussagekraft des Berichts BöckLI, SZW 2016, 444, 460; vgl. auch die allgemeine Kritik an Fairness Opinions bei Beвchuк/Kahan, Duke Law Journal 1989, $27,29 \mathrm{ff}$. 
Ein Fairness Report des zustimmungspflichtigen Aufsichtsrats wäre dagegen weniger kostenintensiv, würde er das Gremium doch lediglich zur frühzeitigen Rechenschaftslegung über seine ohnehin getroffene Entscheidung verpflichten. ${ }^{70}$ Vor dem Hintergrund der Prüfung des Abhängigkeitsberichts durch den Aufsichtsrat gemäß $\ 314 \mathrm{AktG}$ ist aber auch ein solcher Report nicht notwendig.

\section{Zustimmungsvorbehalt des Aufsichtsrats bzw. Related-Party-Ausschusses}

Dem Grundsatz der Organadäquanz folgend ist zu erwarten, dass der deutsche Gesetzgeber die Zustimmungskompetenz für RPT an den Aufsichtsrat vergeben wird. Hinreichend hohe Wesentlichkeitsschwellen vorausgesetzt, würde diese Regelung zu einer lediglich geringfügigen Verschiebung im Kompetenzgefüge zwischen Vorstand und Aufsichtsrat führen.

Auch der mögliche Stimmrechtsausschluss beteiligter related parties und ihrer Repräsentanten wird für Aktionäre vermutlich nicht umgesetzt werden. Für Rechtsgeschäfte der Gesellschaft mit Aufsichtsratsmitgliedern gilt zwar analog $\$ 34$ BGB bereits ein Stimmverbot des betroffenen Mitglieds im Aufsichtsrat. ${ }^{71}$ Ein allgemeines Stimmverbot bei Interessenkonflikten kennt das Gesetz jedoch nicht. ${ }^{72}$ Insbesondere besteht kein Stimmverbot, wenn das Aufsichtsratsmitglied die Gesellschaft beherrscht, ebenso wenig für Repräsentanten des beherrschenden Aktionärs. ${ }^{73}$ Im Aufsichtsrat der faktisch konzernierten AG hat der Gesetzgeber Interessenkonflikte sogar gezielt hingenommen, dienen die abhängigen Aufsichtsratsmitglieder des beherrschenden Aktionärs doch auch der Einbindung in den Konzern. ${ }^{74}$ Für quasi-paritätisch mitbestimmte Gesellschaften ist diezu erwartende Wahl der Ausnahme nach Art. 9c Abs. 4 Satz 4 ARRL II gar unerlässlich: Schon der Stimmrechtsausschluss eines einzigen Anteilseignervertreters würde sich auf die Balance zwischen Anteilseigner- und Arbeitnehmerbank auswirken.

70 Freilich bliebe der Aufsichtsrat in der Pflicht, ggf. zu seiner Information benötigten außenstehenden Sachverstand heranzuziehen; dazu LutTER/Krieger/Verse, Rechte und Pflichten des Aufsichtsrats, 6. Aufl., 2014, Rdn. 245 b.

71 Lutter/Krieger/Verse, aaO (Fn. 70), Rdn. 730.

72 Habersack, Münchener Komm. z. AktG, 4. Aufl., 2014, $\mathbb{1 0 8}$ Rdn. 29; Lutter/KrieGER/Verse, aaO (Fn. 70), Rdn. 730.

73 Habersack, aaO (Fn. 72), $\mathbb{} 108$ AktG Rdn. 30; Hopt/Roth, Großkomm. z. AktG, 4. Aufl., 2017, $\mathbb{1} 108 \mathrm{Rdn} .54$.

74 Vgl. Hommelhoff, ZIP 2013, 1645, 1646 („Rolle des Konzerntransformators“); LUTTER/ Krieger/Verse, aaO (Fn. 70), Rdn. 26. 
Mit den beiden Staatenwahlrechten wurde Art. 9c Abs. 4 ARRL II im Laufe des Gesetzgebungsprozesses allerdings nur partiell entschärft. Der Bruch mit dem Mehrheitsprinzip ist geblieben. Gegen die Mehrzahl der unabhängigen Aufsichtsratsmitglieder wird keine RPT genehmigt werden können, seien die übrigen Aufsichtsratsmitglieder auch in noch so hoher Überzahl. Der Vorschlag der Änderungsrichtlinie, zur Umsetzung qualifizierte Mehrheitserfordernisse für RPT-Abstimmungen vorzusehen, ${ }^{75}$ erscheint im Kontext der deutschen Mitbestimmung wenig geeignet. Angesichts der geringen Zahl unabhängiger Aufsichtsratsmitglieder in konzernierten oder familienkontrollierten Aktiengesellschaften müsste der Gesetzgeber die erforderliche Mehrheit übermäßig hoch ansetzen und würde damit auch den Vertretern der Arbeitnehmer eine Sperrminorität einräumen. Dem gegenüber wäre ein alleiniges Vetorecht für die Mehrheit der unabhängigen Mitglieder eine Durchbrechung des - mit Ausnahme von $\$ 29$ Abs. 2 MitbestG - ehernen Gleichheitsgrundsatzes im Aufsichtsrat. ${ }^{76}$

Bedenkenswert erscheint daher die Übertragung der Zustimmungsentscheidung auf einen Aufsichtsratsausschuss. Teile des Schrifttums sprechen sich seit Längerem dafür aus, die Zustimmung von RPT nach dem Vorbild USamerikanischer special committees einem Ausschuss ganz oder mehrheitlich unabhängiger Aufsichtsratsmitglieder zu überlassen. ${ }^{77}$ Die Richtlinie sieht die Kompetenzübertragung auf ein solches Gremium für den Zustimmungsvorbehalt - im Gegensatz zur Fairness Opinion ${ }^{78}$ - nicht ausdrücklich vor. Im deutschen Recht ist dies gleichwohl kein Hindernis. ${ }^{79}$ Der Aufsichtsratsausschuss ist hier kein eigenes Gesellschaftsorgan, sondern nur besondere Erscheinungsform des Aufsichtsrats. ${ }^{80}$ Im Falle der Aufgabenübertragung handelt der Ausschuss als Aufsichtsrat. ${ }^{81}$ Das Plenum wird hierdurch von seinen Pflichten auch nicht entlastet, sondern muss die Ausschussarbeit nunmehr überwachen und koordinieren. ${ }^{82}$ Vor diesem Hintergrund spricht die Richtlinie zumindest nicht dagegen, es den Aufsichtsräten selbst zu überlassen, ob sie die erforderliche Vetomacht der Unabhängigen über eine fast ausschließ-

75 Siehe Erwägungsgrund 43 der Änderungsrichtlinie (Fn. 2).

76 Dazu Hoffmann-Becking, Münchener Handbuch des Gesellschaftsrechts, Band IV, 4. Aufl., 2015, \33 Rdn. 1 f; Lutter/Krieger/Verse, aaO (Fn. 70), Rdn. 821 m. w. N.

77 Rhiel, Related-Party Transactions im deutschen und US-amerikanischen Recht der Aktiengesellschaft, 2015, S. 211 ff; Rотн, ZHR 175 (2011), 605, $632 \mathrm{f}$.

78 Art. 9 c Abs. 3 lit. c ARRL II.

79 A.A. Tröger, AG 2015, 53, 70f, zu den insoweit gleichlautenden Vorschlägen der italienischen Ratspräsidentschaft.

80 Hopt/Roth, aaO (Fn. 73), $\$ 107$ AktG Rdn. 240; Janberg, AG 1966, 1, 3.

81 Semler, AG 1988, 60, 64.

82 Hорт/Roth, aaO (Fn. 73), $\$ 107$ AktG Rdn. 449 f. 
liche Besetzung des Plenums mit unabhängigen Mitgliedern oder einen entsprechend besetzten RPT-Ausschuss sicherstellen. ${ }^{83}$

Manch Aufsichtsrat einer faktischen Konzerntochter oder Familiengesellschaft dürfte nicht einmal die in einem Dreier-Ausschuss erforderliche Mindestzahl von zwei unabhängigen Mitgliedern aufweisen können. Hommelhoff hat eingehend auf die Herausforderungen hingewiesen, vor die ein Unabhängigkeitspostulat solche Gesellschaften im Zusammenspiel mit der unternehmerischen Mitbestimmung stellt. ${ }^{84}$ Der von ihm zur Positionierung aufgerufene Gesetzgeber hat sich inzwischen geäußert, wenn auch mit dünner Begründung. In Ausübung seines Mitgliedstaatenwahlrechts nach Art. 39 Abs. 5 der reformierten Abschlussprüferrichtlinie ${ }^{85}$ hat der Gesetzgeber im $\mathrm{AReG}^{86}$ auf die Voraussetzung der Unabhängigkeit für Mitglieder des Prüfungsausschusses verzichtet und diese sogleich auch für den Finanzexperten nach $\mathbb{} 100$ Abs. 5 AktG gestrichen. In der Begründung des Regierungsentwurfs heißt es dazu, dass „durch die institutionelle Trennung des Prüfungsausschusses von der Geschäftsleitung [...] bereits ein allgemein hohes Maß an Unabhängigkeit sichergestellt" sei. ${ }^{87}$ Inwiefern diese institutionelle Trennung eine hinreichende Unabhängigkeit von Kontrollaktionären zu sichern vermag, wird nicht erörtert. Aktuell enthält nur noch Ziffer 5.4.2 des DCGK die Empfehlung, dass dem Aufsichtsrat eine „nach seiner Einschätzung angemessene Anzahl unabhängiger Mitglieder" angehören solle.

Art. 9c Abs. 4 ARRL II zwingt den Gesetzgeber nun wenige Monate nach der Reform zur „Rolle rückwärts“: Ihm bleibt die Wahl zwischen der systemwidrigen Übertragung der Zustimmungskompetenz auf die Hauptversammlung oder der Vergabe an den Aufsichtsrat - letzteres nur bei hinreichender Stimmmacht unabhängiger Aufsichtsratsmitglieder. ${ }^{88}$ Börsengesellschaften, die ihr einziges unabhängiges Aufsichtsratsmitglied nach der Reform des $\$ 100$ Abs. 5 AktG gerade erst durch einen abhängigen Finanzexperten ersetzt haben, werden in Folge der Umsetzung der kommenden Richtlinie gleich zwei Posten mit

83 Dazu Spindler/Seidel, AG 2017, 169, 173 ff, die zur Erfüllung der Umsetzungspflicht Deutschlands jedoch eine verpflichtende gesetzliche Regelung zur Ausschussbildung als notwendig erachten.

84 Hommelhoff, ZIP 2013, 953, 955 ff; ders., ZIP 2013, 1645, $1647 \mathrm{ff}$.

85 Siehe Richtlinie 2014/56/EU des Europäischen Parlaments und des Rates vom 16.4.2014 zur Änderung der Richtlinie 2006/43/EG über Abschlussprüfungen von Jahresabschlüssen und konsolidierten Abschlüssen, ABl. L 158 vom 27.4.2014, S. 196.

86 Abschlussprüfungsreformgesetz, BGBl. I 2016, S. 1142; dazu SchÜppen, NZG 2016, 247.

87 Begr. RegE AReG, BT-Drucks. 18/7219, S. 56; kritisch dazu Hommelhoff, in: Habersack/Behme/Eidenmüller/Klöhn, Deutsche Mitbestimmung unter europäischem Reformzwang, 2016, S. 6, 17 f; Strenger, ACI Quarterly I/2016, 42 f; Velte, WPg 2015, $482,488 \mathrm{f}$.

88 Vgl. Bayer/Selentin, NZG 2015, 7, 13. 
Unabhängigen neu besetzen müssen. Der Einwand, man erfülle die Vorgaben ohnehin, da sämtliche Entscheidungen mangels unabhängiger Aufsichtsratsmitglieder ohne gegenteilige Meinung derselben gefasst würden, dürfte jedenfalls nicht durchgreifen. Art. 9c Abs. 4 ARRL II setzt einen adäquaten Minderheitenschutz in der Abstimmung voraus.

\section{Keine Berücksichtigung des Gruppeninteresses, Abschaffung des gestreckten Nachteilsausgleichs}

Da schon die Veröffentlichungspflichten in Art. 9c ARRL II ausschließlich auf die Fairness und Vernünftigkeit einer RPT abzielen, wird sich der Aufsichtsrat bei seiner Zustimmungsentscheidung ebenfalls an diesen Kriterien zu orientieren haben..$^{89}$ Damit stellt sich im Konzern die Frage, ob das Gruppeninteresse berücksichtigt werden darf. Art. 9c ARRL II verweist in sämtlichen seiner Verhaltenspflichten lediglich auf die Belange der Gesellschaft sowie der außenstehenden Aktionäre. Das Interesse der Gruppe darf bei einer Transaktion mit anderen Konzerngesellschaften daher nur insoweit berücksichtigt werden, wie es sich mit den Interessen dieser Schutzadressaten deckt. Eine im Allgemeinen ausgewogene Konzernpolitik unter Berücksichtigung aller Gruppengesellschaften kann die Zustimmung zu nachteiligen Einzelgeschäften nicht rechtfertigen.

Für das Recht des faktischen Konzerns sieht $\$ 311$ AktG dagegen einen strikten Nachteilsausgleich innerhalb eines Geschäftsjahrs vor, der in Verbindung mit den in $\$ \mathbb{S} 312 \mathrm{ff}$ AktG vorgesehenen Kontroll- und Sanktionsmechanismen sichergestellt wird. Vor diesem Hintergrund wird erwogen, ob eine mit Abschluss der RPT eingegangene, nach dem System der $\$ 311 \mathrm{ff}$ AktG ohnehin gesetzlich bestehende Verpflichtung auf Einräumung eines konkreten Nachteilsausgleichsanspruchs innerhalb eines Geschäftsjahres hinreichend ist. ${ }^{90}$

Wenngleich das System der $\mathbb{S} 311 \mathrm{ff}$ AktG insgesamt dem Außenseiterschutz dient, so besteht weitgehende Einigkeit, dass die Streckung des Nachteilsausgleichs eine Privilegierung der Konzernobergesellschaft ist. ${ }^{91}$ Sie erlaubt, den Vorstand der Konzerntochter zunächst ausgleichslos zu Maßnahmen zu veranlassen, die ein ausschließlich mit Blick auf das Interesse der Tochtergesellschaft handelnder ordentlicher Geschäftsleiter nicht ausführen wür-

89 J. VetTER, ZHR 179 (2015), 273, 312.

90 Vgl. J. VeTTER, ZHR 179 (2015), 273, 313.

91 Vgl. BGHZ 179, 71, 78 Rdn. 12; HabersaCK, in: Emmerich/Habersack, Aktien- und

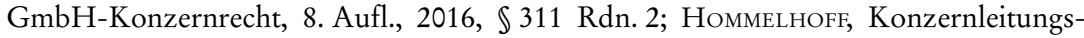
pflicht, 1982, S. 124 f; Koch, in: Hüffer/Koch, Komm. z. AktG, 12. Aufl., 2016, $\$ 311$ Rdn. 4; Koppensteiner, Kölner Komm. z. AktG, 3. Aufl., 2004, Vorb. \311 Rdn. 5. 
de. ${ }^{92}$ Für eine solche Privilegierung lässt das vornehmlich am Fremdvergleichsmaßstab zu messende Fairness-Kriterium kein Raum. Für nachteilige RPT gilt daher gemäß der kommenden Richtlinie, dass spätestens mit Abschluss des Geschäfts festgelegt werden muss, wann und durch welche Vorteile der Nachteil ausgeglichen werden soll. Die Möglichkeit der zeitversetzten Bestimmung bleibt aus. ${ }^{93}$ Damit entfällt für den Bereich der RPT ein Element des faktischen Konzernrechts, dessen Abschaffung ${ }^{94}$ oder restriktive Handhabung ${ }^{95}$ von Teilen des Schrifttums ohnehin seit Langem gefordert wird. Eine Erosion des Ausgleichssystems der $\mathbb{S} 311 \mathrm{ff}$ AktG infolge dieser Änderung ist jedenfalls nicht zu befürchten, geht sie doch mit der einschränkenden Auslegung des $₫ 311$ Abs. 2 AktG in der jüngeren BGH-Rechtsprechung konform. ${ }^{96}$ Auch in der Praxis der Unternehmen hat die Streckung des Nachteilsausgleichs keine große Bedeutung. ${ }^{97}$

\section{Gelockerte Publizitätspflichten für RPT der Tochtergesellschaften}

Die auf Publizitätspflichten beschränkten Bestimmungen des Art. 9c Abs. 7 ARRL II haben weitreichende Auswirkungen im Konzern: Sie können den Kreis der betroffenen Transaktionen je nach Höhe der Wesentlichkeitsschwelle beträchtlich erweitern und verpflichten die börsennotierte Obergesellschaft zur Beobachtung der wesentlichen Geschäfte in allen Tochtergesellschaften. Letztlich bedeuten sie eine weitere Konkretisierung konzerndimensionaler Überwachungspflichten. Ein ebenso hohes Maß an Überwachung wie in der Muttergesellschaft will der europäische Gesetzgeber den Konzernen auf der Tochterebene allerdings nicht zumuten: So bezieht die Aggregationsregelung nach Art. 9c Abs. 8 ARRL II Geschäfte von Tochtergesellschaften nicht ein. Zudem lässt Art. 9c Abs. 7 ARRL II offen, wann die Bekanntmachung von Geschäften der Tochter erfolgen und welche Informationen sie enthalten muss. Der deutsche Gesetzgeber könnte sich demnach auf eine bloße periodische Berichterstattungspflicht ohne begleitende Fairness Opinion beschränken, welche den bezweckten Schutz vor Umgehungen hinreichend erfüllen dürfte.

92 Vgl. Habersack, aaO (Fn. 91), \311 Rdn. 5 („punktuelle Überlagerung ihres Eigenwillens durch einen fremden Willen").

93 So auch U. H. SCHNEIDER, EuZW 2014, 641, 642.

94 Vgl. Hommelhoff, aaO (Fn. 56), G 49; KropfF, FS Kastner, 1992, S. 279, $286 \mathrm{ff}$.

95 So Altmeppen, Münchener Komm. z. AktG, 4. Aufl., 2015, \311 Rdn. 369; Altmeppen, ZIP 2016, 441, $443 \mathrm{ff}$.

96 BGH AG 2012, 680, 682 Rdn. 23; dazu Wilhelm, NZG 2012, 1287.

97 Dazu J. Vetter, aaO (Fn. 56), S. 231, 252. 


\section{Ausschöpfung der optionalen Bereichsausnabmen, feblende Konzernausnabme}

Neben den geringeren Verfahrensanforderungen für RPT im ordentlichen Geschäftsgang sind die zahlreichen Bereichsausnahmen der Richtlinie zu begrüßen. Ihre Wahrnehmung hätte die Union gar nicht erst in die Hände der Mitgliedstaaten beziehungsweise ihrer Unternehmen legen müssen, sind sie doch sämtlich evident sinnvoll.

Für die Praxis der Konzerne ist die Ausnahme von Geschäften mit Tochtergesellschaften nach Art. 9c Abs. 6 lit. a ARRL II sicherlich die bedeutendste Ausnahmeoption. Angesichts der differenzierten Ausgleichs- und Schutzsysteme im deutschen Recht des Vertrags- und faktischen Konzerns wird man von einer Gewährleistung des vorausgesetzten Alternativschutzes im nationalen Recht ausgehen dürfen, sodass der Gesetzgeber sämtliche downstreamTransaktionen einer Konzernmuttergesellschaft vom Anwendungsbereich des Art. 9c ARRL II ausnehmen kann. ${ }^{98}$ Die zahlreichen börsennotierten TochterAG in Deutschland ${ }^{99}$ werden dagegen nicht verschont werden: UpstreamTransaktionen einer börsennotierten Gesellschaft sowie Geschäfte mit Schwestergesellschaften unterliegen uneingeschränkt den kommenden Publizitäts- und Zustimmungspflichten. Die von Praktikern und Verbänden nachdrücklich geforderte vollständige Ausnahme von Konzernsachverhalten ${ }^{100}$ sieht die Richtlinie nicht vor.

Für das deutsche Recht wäre insbesondere eine Ausnahme des Vertragskonzerns sinnvoll gewesen. ${ }^{101}$ Mit der finalen Fassung ist dagegen klar: Die börsennotierte Vertragstochter hat bald keinen Zweck mehr. Ein verbreitetes Phänomen war sie zwar ohnehin nie, spielte im Rahmen öffentlicher Übernahmen aber eine wichtige Rolle. ${ }^{102}$ Mit Abschluss eines Beherrschungs- und Gewinnabführungsvertrags konnten Zielgesellschaften schon vor einem etwaigen Squeeze-out stärker in den Konzern der übernehmenden Gesellschaft

98 Zum entsprechenden Schutzinstrumentarium des deutschen Rechts BAYER/SELENTIN, NZG 2015, 7, 10 f; Fleischer, BB 2014, 2691, $2696 \mathrm{ff}$; Selzner, ZIP 2015, $755 \mathrm{ff}$; J. VetTer, ZHR 179 (2015), 273, 289 ff; WiersCH, NZG 2014, 1131, 1134f; ausführlich für den faktischen Konzern Rhiel, aaO (Fn. 77), S. 23 ff.

99 Beispiele bei Hommelhoff, ZIP 2013, 1645, 1649; ihre Zahl dürfte sich angesichts des anhaltenden Trends zu Teilbörsengängen deutscher Konzerntöchter noch erhöhen, vgl. KÖHLER/LANDgraf, Handelsblatt vom 11.7.2017, S. 1, 4 f.

100 Vgl. etwa BDA/DAI Positionspapier vom 12.11.2015, abrufbar unter https://www.dai. de/files/dai_usercontent/dokumente/positionspapiere/2015-11-12\%20SHRD-Position \%20BDI\%20und\%20DAI.pdf; Bungert/De RAET, Der Konzern 2015, 289, 294; SelZNER, ZIP 2015, 753, 760.

101 Diese fordernd Bayer/Selentin, NZG 2015, 7, 12; Habersack, AG 2016, 691, 696; J. VetTER, ZHR 179 (2015), 273, 316; WIERsCh, NZG 2014, 1131, 1134.

102 Vgl. Selzner, ZIP 2015, 753, 760 Fn. 80; J. Vetter, ZHR 179 (2015), 273 , 316. 
integriert werden, als dies das faktische Konzernrecht zuließe. ${ }^{103}$ Wird das weite Feld der RPT der Befugnis zu nachteiligen Weisungen gemäß $\$ 308$ Abs. 1 Sätze 1, 2 AktG nun entzogen und Veröffentlichungs- und Zustimmungspflichten unterworfen, entfällt dieser Vorteil. Künftig werden tiefgreifende Konzernintegrationsmaßnahmen erst nach einem Rückzug der Tochtergesellschaft von der Börse möglich sein. $\mathrm{Ob}$ das Vertragskonzernrecht vor diesem Hintergrund als insgesamt "gerettet“ gelten kann, ${ }^{104}$ bedarf kritischer Analyse. Zur Veranschaulichung der Problematik sei folgendes Beispiel genannt: ${ }^{105}$

Der US-Konzern Procter \& Gamble erwarb im Jahr 2003 von den Familienaktionären der Darmstädter Wella AG rund $78 \%$ der stimmberechtigten Aktien. Die angestrebte Übernahme sämtlicher Anteile wurde erst Ende 2007 per Squeeze-out der verbliebenen Minderheitsaktionäre erreicht. Anfechtungsklagen gegen den entsprechenden Hauptversammlungsbeschluss vom 13./14. Dezember 2005 hatten die Übernahme um fast 2 Jahre verzögert. Eine weitgehende Integration in den Procter \& Gamble-Konzern war gleichwohl schon durch den 2004 geschlossenen Beherrschungs- und Gewinnabführungsvertrag zwischen der Wella AG und der Procter \& Gamble Holding $\mathrm{GmbH} \&$ Co. Operations oHG möglich. Ob dies auch unter Geltung des neuen RPT-Regimes möglich gewesen wäre, darf bezweifelt werden: Wesentliche Leistungen der übernommenen Wella AG an die Konzernmutter sowie Schwestergesellschaften im Procter \& Gamble-Konzern hätten ungeachtet der abgeschlossenen Unternehmensverträge einer Veröffentlichung und Hauptversammlungs- bzw. Aufsichtsratszustimmung am Maßstab des fairness-Kriteriums bedurft.

Mit der möglichen Ausnahme von bereits nach nationalem Recht der Hauptversammlung vorzulegenden Geschäften gemäß Art. 9c Abs. 6 lit. b ARRL II kann der deutsche Gesetzgeber insbesondere Strukturmaßnahmen von den neuen Verfahrenspflichten befreien. Ihnen ist im deutschen Recht gemein, dass sie von der Hauptversammlung zu beschließen sind und, neben jeweiligen spezifischen Schutzinstrumenten, über das Erfordernis einer qualifizierten Mehrheit ein übergreifendes Mindestmaß an Minderheitenschutz gewährleistet ist. ${ }^{106}$ Naheliegende Anwendungsbereiche für den Gebrauch der Ausnahmeoption sind daher unter anderem Unternehmensverträge, Umwandlungsmaßnahmen und Bezugsrechtsemissionen.

Da Art. 9c Abs. 6 lit. b ARRL II kein Stimmverbot der nahestehenden Person voraussetzt, muss der deutsche Gesetzgeber für die AG auch künftig kein $\ 47$

103 J. VetTER, ZHR 179 (2015), 273, 316.

104 So J. Schmidt, Der Konzern 2017, 1, 10.

105 Vgl. die Ausführungen zum Tatbestand bei BGHZ 189, 261, $262 \mathrm{f}$.

106 K. SснміDт, Gesellschaftsrecht, 4. Aufl., 2002, S. 919. 
Abs. 4 GmbHG beziehungsweise $\ 252$ Abs. 3 HGB a.F. vergleichbares umfassendes Stimmverbot für Rechtsgeschäfte mit Gesellschaftern (wieder-)einführen. Insbesondere wird der Mehrheitsaktionär entsprechend der bewussten Entscheidung des Gesetzgebers von 1965 weiterhin an Zustimmungsbeschlüssen der Hauptversammlung zu Unternehmensverträgen mitwirken können. ${ }^{107}$

\section{Keine Anwendung auf geschlossene Gesellschaften}

Auch die reformierte Aktionärsrechterichtlinie beschränkt sich auf eine Harmonisierung des Rechts börsennotierter Gesellschaften. Im Hinblick auf die RPT-Regelungen ist im Schrifttum eine überschießende Umsetzung für börsenferne Gesellschaften erwogen worden. ${ }^{108}$ In der Tat bergen RPT in geschlossenen Gesellschaft dieselben Gefahren wie in Börsengesellschaften, zumal erstere mehrheitlich durch konzentrierten Anteilsbesitz geprägt sind. Nach Durchsicht des Art. 9c ARRL II muss jedoch resümiert werden: Die aufwendige Einrichtung des zur Erkennung und Einhaltung der Verfahrenspflichten erforderlichen Kontrollsystems würde sie übermäßig hart treffen. Gerade für kleine und mittlere Unternehmen wären ein Unabhängigen-Ausschusses im Aufsichtsrat und die ständige Kontrolle aller RPT nicht nur ein bürokratisches Ärgernis, sondern auch eine erhebliche finanzielle Belastung. Soll auch für die geschlossenen Gesellschaften ein spezifischer RPT-Schutz geregelt werden, so empfiehlt sich daher eine gelockerte, auf Publizitätspflichten beschränkte Kontrolle. In jedem Fall sollte der Gesetzgeber eine Ausnahme für geschlossene Gesellschaften im Vertragskonzern vorsehen, will er das Institut des Unternehmensvertrags nicht vollständig zu Grabe tragen.

\section{Fazit und Ausblick}

Mit der Umsetzung von Art. 9c ARRL II wird die viel kritisierte zurückhaltende Haltung Deutschlands bei der Kontrolle von related party transactions enden. ${ }^{109}$ Stattdessen werden auch hierzulande spezifische und weit-

107 Vgl. KropfF, Aktiengesetz, 1965, S. 380 f.

108 Vgl. Fleischer, ZGR 2017, 1, 34; Hommelhoff, KSzW 2014, 63, 66; J. VetTer, ZHR 179 (2015), 273, 324; TRÖGER, AG 2015, 53, 61; gegen eine Beschränkung auf börsennotierte Gesellschaften auch die European Company Law Experts (ECLE), A proposal for reforming group law in the European Union, October 2016, abrufbar unter https:// europeancompanylawexperts.wordpress.com/papers_trashed/reforming-group-lawin-the-eu/.

109 Vgl. Enriques/Hertig/Kanda/Pargendler, in: Kraakman et al., The Anatomy of Corporate Law, 3. Aufl., 2017, S. 145, $166 \mathrm{ff}$. 
gehend lückenlose Kontrollmechanismen für diese Geschäfte eingeführt werden.

Für Unternehmensgruppen mit hohem internen Leistungs- und Lieferverkehr bedeuten die neuen Regelungen vor allem einen großen bürokratischen Mehraufwand. Ohne die Einrichtung eines ständigen Kontrollsystems werden sie die Erkennung und Einhaltung der neuen RPT-Pflichten nicht gewährleisten können. Vor diesem Hintergrund kann schon jetzt festgehalten werden: Der von Lutter befürchtete RPT-Beauftragte ${ }^{110}$ wird kommen.

Darüber hinaus fördert die neue EU-Regelung mit der Stärkung der Rolle unabhängiger Mitglieder in Verwaltungs- beziehungsweise Aufsichtsorganen eine weitere internationale Entwicklung, der sich der deutsche Gesetzgeber in jüngerer Zeit bewusst versperrt hat. Vergibt er nun die Zustimmungskompetenz für RPT an den Aufsichtsrat, wird für die meisten Gesellschaften einzig die Bildung eines beschließenden Unabhängigen-Ausschusses im Aufsichtsrat praktikabel sein.

Neben der faktischen Führung deutscher Konzerne bleibt auch das sie regelnde Konzernrecht nicht unangetastet von der neuen Richtlinie. Die vorausgesetzte Fairness jeder Einzeltransaktion lässt keine Ausnahmen wie das deutsche Konzernprivileg des $₫ 311$ Abs. 2 AktG zu. Für den Bereich der RPT fällt eine zeitliche Streckung der Nachteilsausgleiche im faktischen Konzern zukünftig weg. Mit dem Recht des Vertragskonzerns sind die neuen RPT-Regelungen schlicht unvereinbar. Auf das Prinzip der Fairness jeder Einzeltransaktion ausgerichtete Publizitäts- und Zustimmungsverfahren kollidieren mit dem Ausgleichssystem der $\$ \mathbb{S} 300 \mathrm{ff} A \mathrm{ktG}$ und machen die vertragskonzernierte Börsentochter insgesamt unattraktiv.

In ihrem Anwendungsbereich schiebt die neue Richtlinie auch flexiblen Ausgleichssystemen im Konzern nach dem Modell der französischen Rozenblum-Doktrin ${ }^{111}$ einen Riegel vor, wie sie derzeit auf europäischer Ebene diskutiert werden. ${ }^{112}$ Die zu entsprechenden Kommissionplänen ${ }^{113}$ konsultierte Informal Company Law Expert Group (ICLEG) konnte sich nur auf eine Anerkennung des Gruppeninteresses bei Einpersonen-Tochtergesell-

110 LutTer, EuZW 2014, 687, 688.

111 Cass. crim. 4. 2. 1985, JCP/E 1985, II, 14614; dazu Falcke, Konzernrecht in Frankreich, 1997, S. $38 \mathrm{ff}$.

112 Vgl. Forum Europaeum on Company Groups, ECFR 2015, 299; Club des Juristes, Towards Recognition of the Group Interest in the European Union?, Juni 2015, S. 19ff, abrufbar unter https://orbilu.uni.lu/bitstream/10993/28103/1/CDJ_Rapports_Groupinterest_UK_June-2015_web.pdf.

113 Siehe Europäische Kommission, Aktionsplan: Europäisches Gesellschaftsrecht und Corporate Governance - ein moderner Rechtsrahmen für engagiertere Aktionäre und besser überlebensfähige Unternehmen, $\operatorname{COM}(2012)$ 740/2, 12.12.2012, S. 17. 
schaften einigen. ${ }^{114}$ Gerade für diese Gesellschaften bietet Art. 9c ARRL II die passenden Ausnahmen vom neuen RPT-Regime. Ob sich die am Gesetzgebungsverfahren beteiligten Akteure auch bewusst waren, wie weit sie der Ausgestaltung des Projekts Anerkennung des Gruppeninteresses mit der neuen Richtlinie vorgreifen, sei dahingestellt.

114 ICLEG, Report on the recognition of the interest of the group, Oktober 2016, S. 40f, abrufbar unter http://ec.europa.eu/justice/civil/files/company-law/icleg_recommendations_interest_group_final_en.pdf. 